\title{
Quantitative Evaluation of Noncovalent Chorismate Mutase-Inhibitor Binding by ESI-MS
}

\author{
Silke Wendt,* Gregor McCombie,† Jürg Daniel, Alexander Kienhöfer, \\ Donald Hilvert, and Renato Zenobi \\ Laboratory of Organic Chemistry, Swiss Federal Institute of Technology (ETH), Zürich, Switzerland
}

\begin{abstract}
Electrospray time-of-flight mass spectrometry was used to quantitatively determine the dissociation constant of chorismate mutase and a transition state analogue inhibitor. This system presents a fairly complex stoichiometry because the native protein is a homotrimer with three equal and independent substrate binding sites. We can detect the chorismate mutase trimer as well as chorismate mutase-inhibitor complexes by choosing appropriate conditions in the ESI source. To verify that the protein-inhibitor complexes are specific, titration experiments with different enzyme variants and different inhibitors were performed. A plot of the number of bound inhibitors versus added inhibitor concentration revealed saturation behavior with 3:1 (inhibitor:functional trimer) stoichiometry for the TSA. The soft ESI conditions, the relatively high protein mass of $43.5 \mathrm{kDa}$, and the low charge state (high $\mathrm{m} / \mathrm{z}$ ) result in broad peaks, a typical problem in analyzing noncovalent protein complexes. Due to the low molecular weight of the TSA $(226 \mathrm{Da})$ the peaks of the free protein and the protein with one, two or three inhibitors bound cannot be clearly resolved. For data analysis, relative peak areas of the deconvoluted spectra of chorismate mutase-inhibitor complexes were obtained by fitting appropriate peak shapes to the signals corresponding to the free enzyme and its complexes with one, two, or three inhibitor molecules. From the relative peak areas we were able to calculate a dissociation constant that agreed well with known solution-phase data. This method may be generally useful for interpreting mass spectra of noncovalent complexes that exhibit broad peaks in the high $\mathrm{m} / \mathrm{z}$ range. (J Am Soc Mass Spectrom 2003, 14, 1470-1476) () 2003 American Society for Mass Spectrometry
\end{abstract}

$\mathrm{W}$ ith the rapid progress in genomic sequencing and proteomics, noncovalent interactions of proteins with their binding partners are becoming a major focus of attention. Especially in drug development, where large libraries of ligands (e.g. inhibitors) are often screened, a facile method for determining relative binding strengths with low substance consumption is desirable.

Soft ionization mass spectrometry, such as electrospray ionization (ESI), is able to provide useful information about noncovalent assemblies involving biological macromolecules, including their complexes with small ligands or inhibitors. Numerous studies have been published on this topic, and a number of good reviews have appeared [1-3]. Increasingly, the question of whether dissociation constants of noncovalent com-

\section{Published online October 30, 2003}

Address reprint requests to Dr. R. Zenobi, Department of Chemistry, Swiss Federal Institute of Technology (ETH), Hönggerberg, HCI E 329, CH-8093 Zürich, Switzerland. E-mail: zenobi@org.chem.ethz.ch

*Current address: GSF-Forschungszentrum für Umwelt und Gesundheit, $\mathrm{GmbH}$, Institut für Toxikologie, Ingolstädter Landstraße 1, D-85764 Neuherberg, Germany.

tCurrent address: Novartis Institutes for Biomedical Research, 4002 Basel, Switzerland. plexes can be determined by mass spectrometry is being addressed (for a review see [4]). Because mass spectrometry employs gas-phase ions, conditions must be found such that the complexes survive the desolvation and ionization processes. If successful, the mass spectrometer can be considered as a detector for solutionphase chemistry. Several methods have already been published where noncovalent solution-phase interactions have been analyzed quantitatively by mass spectrometry, including mass spectrometrically detected melting curves [5], competition studies [6-8], and titration experiments [9-14]. Only a small number of studies have appeared in the literature reporting the quantitative investigation of protein inhibition by mass spectrometry [6, 8-10]. Among these, the studies by Greig et al. [10] and Ayed et al. [11] involved interactions with larger proteins/enzymes.

The most obvious advantages of mass spectrometry include the possibility of obtaining the mass and stoichiometry of a complex directly, short measurement times, and the small amount of analyte required. It could represent a useful method for determining binding constants of mutant proteins, where classical activity measurements cannot distinguish between residual 
activity of the mutant and activity due to wild type enzyme still present as an impurity. A difficulty of mass spectrometry using very soft ionization is that adducts from solvent and/or buffer can survive the ionization process and broaden the signals such that it becomes difficult or impossible to distinguish small ligand adducts in the data. We addressed this problem by a new data evaluation procedure.

The system studied here is the enzyme chorismate mutase (E.C. 5.4.99.5) from Bacillus subtilis (BsCM), a model for protein assembly as well as ligand binding. $\mathrm{BsCM}$ catalyzes the rearrangement of chorismate to prephenate, the first committed step in the biosynthesis of the aromatic amino acids phenylalanine and tyrosine. Since chorismate mutases are found in bacteria, fungi, and higher plants, designing and screening inhibitors for this enzyme might be of importance for the development of new herbicides and antibacterial therapeutics. The structure and function of BsCM are well characterized. The crystal structure shows a homotrimeric pseudo- $\beta$-barrel surrounded by $\alpha$-helices with three solvent-accessible binding sites at the subunit interfaces [15]. As has been shown in solution the three binding sites are all equal and independent [16]. A BsCM monomer has a molecular weight of $14,489 \mathrm{Da}$, leading to a molecular weight of $43,467 \mathrm{Da}$ for the functional trimer. Throughout this paper, the abbreviation $\mathrm{BsCM}$ represents the functional trimeric enzyme. Several inhibitors have been reported for chorismate mutases, including a transition state analogue (TSA) designed by Bartlett and coworkers [17], which is used in this study. This system allows us to investigate the stability of the noncovalent trimer as well as the stability of BsCM-inhibitor complexes in the gas phase.

\section{Experimental}

\section{Instrumentation}

All experiments were conducted on a prototype electrospray orthogonal injection time-of-flight mass spectrometer from Agilent (Palo Alto, CA). It consists of a commercial electrospray interface (Agilent), two skimmers, and an octopole ion guide for collisional cooling. The ions are extracted orthogonally by a pulsed extraction field at a repetition rate of $4 \mathrm{kHz}$. The ions are guided via a single stage reflectron to the detector (ETP Electron Multipliers Pty Ltd. Ermington, Australia). The detector signal is amplified and digitized with a computer (Hewlett Packard, Palo Alto, CA) at a sampling frequency of $250 \mathrm{MHz}$. The mass resolution of the instrument is around 1100 in the mass range employed here. Infusion rates of 1 to $5 \mu \mathrm{l} / \mathrm{min}$ with a pneumatically-assisted ESI source were used. The spray potentials were between 2700 and $3000 \mathrm{~V}$. The source conditions of the instrument were optimized for the transfer of high molecular weight ions and for gentle desolvation conditions by adjusting the different source and transfer potentials. Spectra were recorded in positive mode with a repetition rate of $4 \mathrm{kHz}$ 1,000,000 transients per spectrum were summed and transferred to a PC for further processing. The potential applied to the exit of the transfer capillary is variable and is referred to $\mathrm{V}_{\mathrm{ex}}$ below; the potential at the first skimmer is $\mathrm{V}_{\mathrm{sk} 1}$.

\section{Chorismate Mutases}

Wild-type BsCM was produced in E. coli and purified as described [18]. The truncated mutant $\mathrm{BsCM}^{1-120}$ was generated by PCR amplification of the gene segment encoding residues $1-120$ of $\mathrm{BsCM}$ using plasmid pKET3-W [19] as the template and T7PRO2 (TAATACGACTCACTATAGGG) and SVSF-7-L121* (AACTCCTCGAGTTAGCTTAAATCGGGCCTCAATA) as the forward and reverse primers, respectively. The nucleotides for the stop codon replacing the triplet encoding Leu121 are shown in bold. The gene fragment was purified, digested with BsrGI and XhoI, and ligated into the acceptor fragment obtained by digesting pKET3-W with the same enzymes. The insert in the resulting plasmid, pAK-L121*, was verified by sequencing using primer 04-T7TR (CAGCAGCCAACTCAGCTTCCTTTC) [20]. The chorismate mutase deficient E. coli strain KA13 [21] transformed with pAK-L121* afforded $40 \mathrm{mg}$ of purified $\mathrm{BsCM}^{1-120}$ per $1 \mathrm{~L}$ of culture. The molecular weight of the mutant protein was confirmed by ESI-MS (experimental result 13,691 \pm 2 Da; calculated 13,689 Da). No impurities were observed by polyacrylamide gel electrophoresis. ESI-MS showed that the N-terminal methionine is partially removed, as is typical for bacterial proteins, but this has no effect on the enzymatic activity. However, it leads to a low-mass shoulder that can be seen in some of the ESI mass spectra presented below.

Prior to ESI-MS measurements, the proteins were desalted three times by gel filtration (NAP-5 column, Amersham Pharmacia Biotech, Uppsala, Sweden). ESI measurements were made with a concentration of 10 $\mu \mathrm{M}$ protein monomer, corresponding to $3.3 \mu \mathrm{M}$ BsCM in the active (trimeric) form, dissolved in 10 to $20 \mathrm{mM}$ triethyl ammonium bicarbonate buffer $(\mathrm{pH} \approx 8)$. Protein concentration was determined by UV spectroscopy prior to desalting. Recovery factors of the desalting columns were $\geq 97 \%$ such that uncorrected values of protein concentration were used.

\section{Inhibitors}

TSA was synthesized according to literature procedures [17]. In addition, adamantyl-1-phosphonate (AP), synthesized according to a published procedure [22], was used as a very weak BsCM inhibitor.

\section{Inhibition Assays}

The enzymes were assayed as previously described [18] at $\mathrm{pH}=7.5$ in $50 \mathrm{mM}$ potassium phosphate buffer or in triethylammonium bicarbonate (TEAB) buffer, respec- 
tively. The $K_{d}$ values were calculated from the slope of the line obtained by plotting [enzyme] $/ \mathrm{v}_{0}$ versus the inhibitor concentration using the equation, $\mathrm{K}_{\mathrm{d}} \mathrm{d}=\mathrm{K}_{\mathrm{m}}$ / $\left(\mathrm{k}_{\text {cat }}{ }^{*}\right.$ [substrate] * slope), where $\mathrm{K}_{\mathrm{m}}$ and $\mathrm{k}_{\text {cat }}$ are the independently measured Michaelis and catalytic rate constants obtained in the absence of inhibitor $\left[k_{\text {cat }}\right.$ $\left(w_{t B s C M}\right)=46 \mathrm{~s}^{-1} ; K_{\mathrm{m}}=100 \mu \mathrm{M} ; \mathrm{k}_{\mathrm{cat}}\left(\mathrm{BsCM}^{1-120}\right)=44$ $\left.\mathrm{s}^{-1} ; \mathrm{K}_{\mathrm{m}}=1010 \mu \mathrm{M}\right]$. The enzyme was used at a concentration of $10 \mathrm{nM}$, the substrate concentration between 25 and $400 \mu \mathrm{M}$ for BsCM and between 20 and $2000 \mu \mathrm{M}$ for $\mathrm{BsCM}^{1-120}$. The inhibitor concentration was varied between 0.5 and $50 \mu \mathrm{M}$ depending on the experimentally determined $K_{d}$ value. $K_{d}$ values obtained by this method are reported per binding pocket, whereas in our analysis of the mass spectrometric data, $K_{d}$ values refer to the concentration of the total trimer concentrations. Thus, the $K_{d}$ values obtained by mass spectrometry were divided by a factor of 3 for comparison with literature and measured solution-phase data.

\section{Results and Discussion}

\section{Detection of the Chorismate Mutase Trimer}

A prerequisite for the detection of specific noncovalent interactions between a protein and bound inhibitors is the preservation of a near-native structure of the protein during passage from solution phase to the gas phase. As the active form of $\mathrm{BsCM}$ is a homotrimer, it is necessary to detect the trimer in the mass spectrum. It has been reported [23] that multiply charged ions generated in TEAB solution are significantly more stable than those formed in conventional buffers such as ammonium bicarbonate or acetate. Our experience has confirmed this observation, and we generally used TEAB at a concentration of 10 to $20 \mathrm{mM}$. Spectrophotometric assays showed that BsCM retains full activity in this buffer. The BsCM trimer was detected at low $\mathrm{V}_{\mathrm{sk} 1}$. With increasing $\mathrm{V}_{\mathrm{sk} 1}$ it dissociated into monomers that are not observed in solution under physiological conditions (Figure 1). However, peaks corresponding to dimers were in no case detected. Likewise no signals for tetramers or pentamers could be observed under any conditions. Both observations speak against formation of unspecific clusters of BsCM monomers, suggesting that specific native-like protein-protein interactions account for the observation of trimeric species at low $\mathrm{V}_{\text {sk1 }}$. Together with the binding constants determined by mass spectrometric methods described later in this work which are comparable to solution-phase binding constants, these findings show that the detected trimer is indeed the result of specific interactions.

\section{Detection of Trimer-Inhibitor Complexes}

To be able to detect a weak noncovalent interaction, very soft conditions in the ESI source must be chosen in order to prevent the complex from dissociating. However, reasonable desolvation of the complexes is still

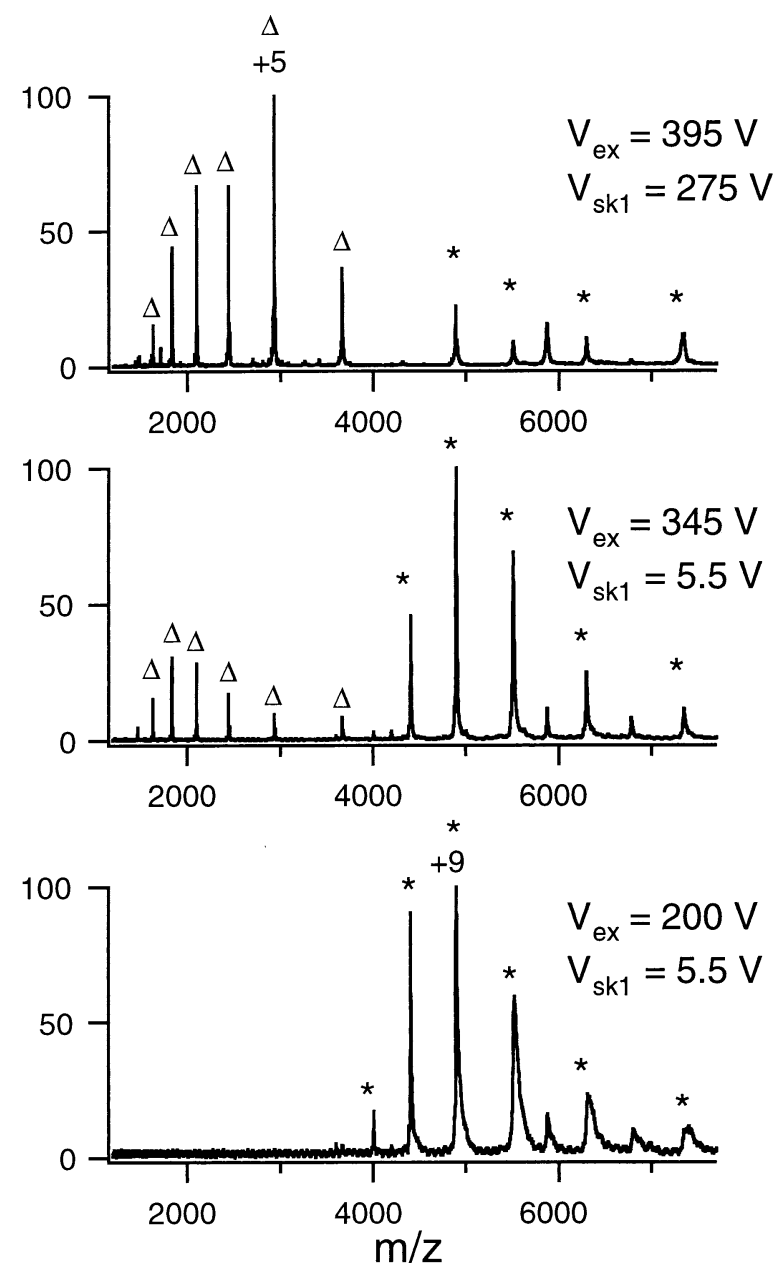

Figure 1. ESI-TOF mass spectra of a solution of $3.3 \mu \mathrm{M}$ BsCM. By decreasing $V_{\mathrm{ex}}$ and $\mathrm{V}_{\mathrm{sk} 1}$ and hence going to softer conditions, peaks corresponding to the trimer (represented by an asterisk) become the only species detected. Peaks corresponding to the monomer (represented by an open triangle) disappear.

necessary to obtain a satisfactory signal-to-noise ratio and to avoid extensive adduct formation. A compromise must thus be found for the operating conditions of the ESI source. Figure 2 shows the $\left[\mathrm{M}+8 \mathrm{H}^{+}\right]^{8+}$ peak of the $\mathrm{BsCM}$ trimer from spectra of a solution $(3.3 \mu \mathrm{M}$ BsCM and $10 \mu \mathrm{M}$ TSA in $10 \mathrm{mM}$ TEAB) recorded at different $V_{\text {ex }}$ and $V_{\text {sk1 }}$ values. At high voltages, corresponding to harsher conditions, the peak intensitiy is very good, and the mass is only slightly larger than the calculated mass of a bare BsCM trimer (Spectrum A). The small mass difference is due to incomplete desolvation of the native trimer and buffer molecule adducts, as previously described [24]. Decreasing $\mathrm{V}_{\mathrm{ex}}$ and $\mathrm{V}_{\mathrm{sk} 1}$, and hence going to softer conditions, gives a clear shift to higher mass. The peak also becomes broader, but shows partial resolution of the different BsCM-inhibitor complexes (Spectrum B). With even softer conditions the peak shifts further in the direction of higher mass (Spectrum C) with the maximum close to the theoretical mass of a BsCM.I ${ }_{3}$ complex, while the resolution of the different complex peaks, however, is lost. Interestingly, 


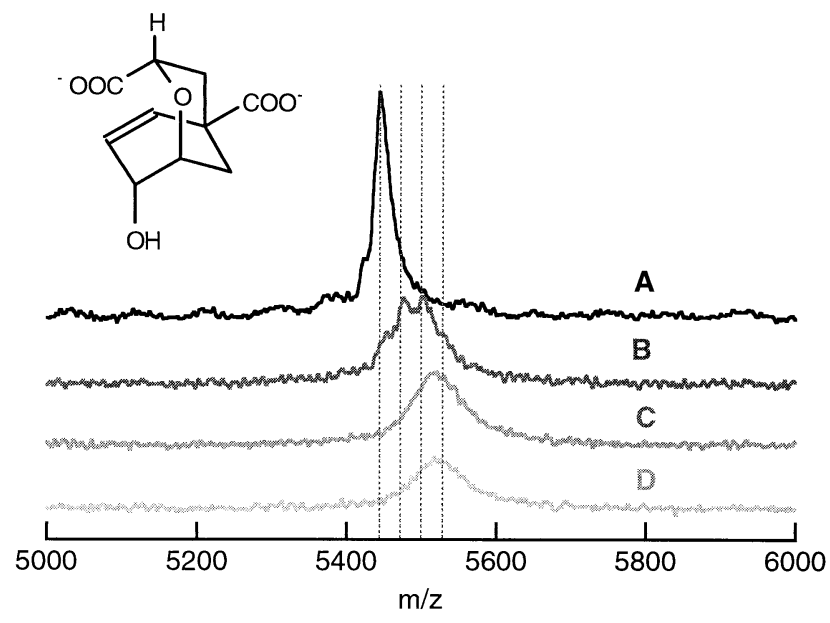

Figure 2. ESI-TOF mass spectra $\left(\left[\mathrm{M}+8 \mathrm{H}^{+}\right]^{+8}\right.$ charge state) of a solution of $3.3 \mu \mathrm{M}$ BsCM and $10 \mu \mathrm{M}$ TSA inhibitor at different desolvation conditions. From top to bottom conditions become increasingly softer: (a) $\mathrm{V}_{\mathrm{ex}}=250 \mathrm{~V}, \mathrm{~V}_{\mathrm{sk} 1}=138 \mathrm{~V}$; (b) $\mathrm{V}_{\mathrm{ex}}=150 \mathrm{~V}$, $\mathrm{V}_{\mathrm{sk} 1}=83 \mathrm{~V}$; (c) $\mathrm{V}_{\mathrm{ex}}=100 \mathrm{~V}, \mathrm{~V}_{\mathrm{sk} 1}=55 \mathrm{~V}$; (d) $\mathrm{V}_{\mathrm{ex}}=50 \mathrm{~V}, \mathrm{~V}_{\mathrm{sk} 1}=$ $28 \mathrm{~V}$. The vertical lines represent the mass of bare $\mathrm{BsCM}$ and the calculated peak positions of $\mathrm{BsCM}$ with one, two and three inhibitors bound. Note the mass shift towards the $\mathrm{BsCM} \cdot \mathrm{I}_{3} \mathrm{com}$ plex as the conditions become softer. The low-mass shoulder is due to partial removal of N-terminal methionine, as is typical for bacterial proteins. Inset: Structure of the TSA inhibitor.

a further decrease in $V_{\text {ex }}$ and $V_{\text {sk } 1}$ does not result in a further shift towards higher mass but only to a loss in signal intensity (Spectrum D). In the following titration experiments we therefore chose the conditions used for spectrum $C$ since under these conditions the complex does not appear to dissociate in the gas phase, while signal intensity is still reasonable. It should be noted that the monomeric enzyme subunit is not evident in any of the spectra shown in Figure 2.

\section{Titration Experiments with Different Inhibitors and Chorismate Mutase Mutants}

Before the binding strength of an inhibitor for an enzyme can be determined, the specificity of the detected complex must be verified. Since the mass is the main observable in mass spectrometry, it cannot be decided from one single measurement whether the inhibitor is located in the binding pocket or simply bound non-specifically to the surface of the protein. To address this question we used a BsCM mutant in addition to wild type BsCM, as well as the much weaker AP inhibitor $\left(\mathrm{K}_{\mathrm{d}}>4 \mathrm{mM}\right)$. The mutant species, called $\mathrm{BsCM}^{1-120}$, was shorter by the last seven Cterminal amino acids in the sequence and exhibited reduced enzyme activity and ligand affinity, as reported previously for other randomly truncated $\mathrm{BsCM}$ mutants [25].

A series of experiments was performed by varying the concentration of the TSA or AP inhibitors in the presence of $3.3 \mu \mathrm{M}$ BsCM or $\mathrm{BsCM}^{1-120}$. Since the peaks corresponding to the free enzyme and its complexes

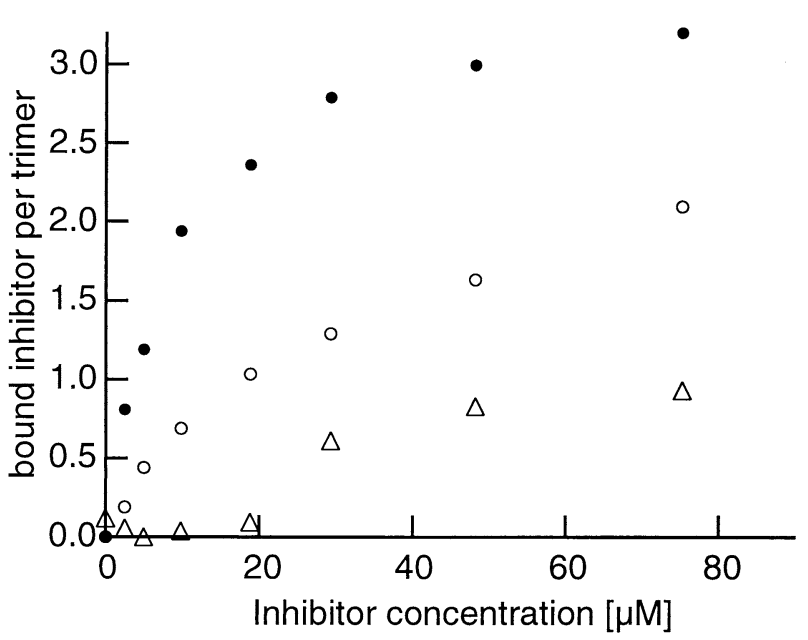

Figure 3. Plot of number of bound inhibitors against inhibitor concentration for TSA / BsCM (filled circles) and TSA / $\mathrm{BsCM}^{1-120}$ (open circles), and $\mathrm{AP} / \mathrm{BsCM}$ (open triangles). BsCM concentration was $3.3 \mu \mathrm{M}$ in all cases. The number of bound inhibitors was determined from the deconvoluted spectra as described in the text.

with one, two or three inhibitor molecules are not clearly resolved, the statistical number of bound inhibitor molecules was determined. Spectra such as those shown in Figure 2 were deconvoluted to represent the +1 charge state only, smoothed, and the average mass at every inhibitor concentration was obtained. The mass difference $\Delta \mathrm{m}$ between this average mass and the mass of the free protein was then divided by the mass of the inhibitor to yield the number of bound inhibitor molecules. In Figure 3, the results for TSA/ BsCM, TSA/ $\mathrm{BsCM}^{1-120}$, and $\mathrm{AP} / \mathrm{BsCM}$ are plotted against the added inhibitor concentration. For wild type BsCM binding TSA, this results in a saturation curve, the limit of which at a 20-fold molar excess of inhibitor slightly exceeds the theoretical maximum of three bound inhibitors. At this excess and under these conditions (low salt concentrations) some nonspecific binding can apparently occur. However, we take the clear saturation behavior at a value of three inhibitors per trimer as evidence for a specific binding to the binding pocket. The curves for TSA/BsCM ${ }^{1-120}$ and for AP/BsCM show a lower increase in binding of inhibitor, which can be attributed to weaker binding.

\section{Calculation of $K_{d}$}

The binding of the inhibitor to $\mathrm{BsCM}$ showed an apparent dependence on the charge state, i.e. peak intensity ratios of complex relative to bare enzyme appeared to be different for every charge state. A straightforward explanation for this observation is a conformational change induced by the inhibitor, which often leads to a change in the charge state distribution in the ESI mass spectra. Consider, for example, a most abundant charge state of $\left[\mathrm{M}+10 \mathrm{H}^{+}\right]^{10+}$ for the naked $\mathrm{BsCM}$ and a maximum charge state of $\left[\mathrm{M}+9 \mathrm{H}^{+}\right]^{9+}$ 


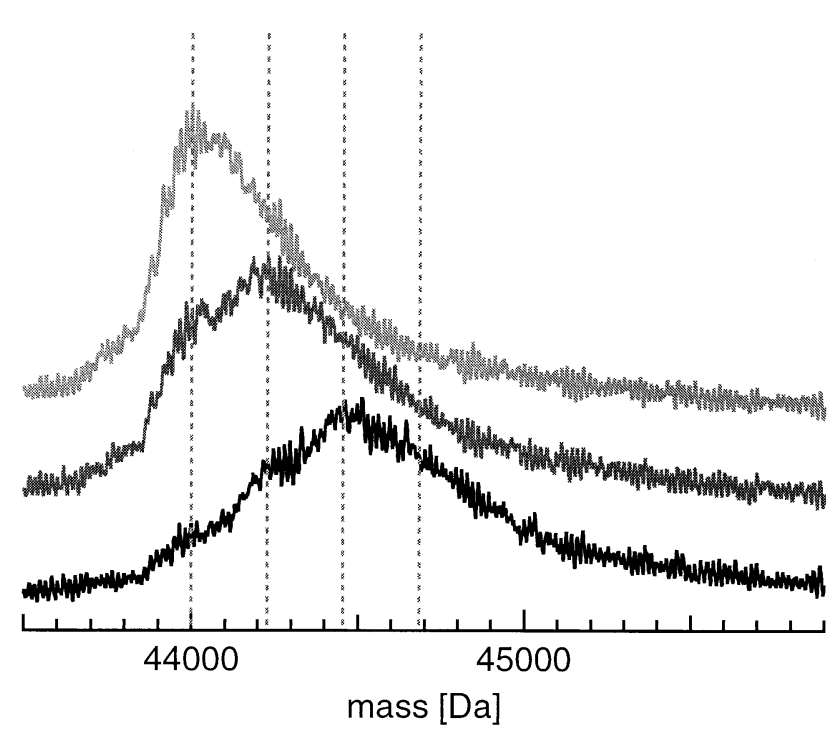

Figure 4. Deconvoluted ESI mass spectra of BsCM (top), BsCM plus $2.5 \mu \mathrm{M}$ TSA (middle) and BsCM plus $10 \mu \mathrm{M}$ TSA (bottom). $\mathrm{BsCM}$ concentration was $3.3 \mu \mathrm{M}$. The vertical lines represent the peak position of bare BsCM determined by ESI-MS and the calculated peak positions of $\mathrm{BsCM}$ with one, two, and three inhibitors bound.

after binding of the inhibitor. An overlay of the two ESI mass spectra will then result in different intensity ratios at every peak, i.e. in an apparent charge state dependent inhibitor binding. The correct way to deal with this is to calculate dissociation constants from the deconvoluted spectrum (representing the charge state $\mathrm{z}=0$, i.e., without any protons attached), thus taking into account all charge states observed in the spectrum.

The resulting deconvoluted peaks of some selected spectra are shown in Figure 4. To evaluate the data quantitatively we need to be able to determine the relative contributions of the free enzyme and the individual complexes to the total peak area, i.e., the ratio of $\mathrm{BsCM} / \mathrm{BsCM} \cdot \mathrm{I}, \mathrm{BsCM} \cdot \mathrm{I} / \mathrm{BsCM} \cdot \mathrm{I} 2$ or $\mathrm{BsCM} \cdot \mathrm{I} 2 / \mathrm{BsCM} \cdot \mathrm{I}_{3}$. Due to large, overlapping peaks, these ratios cannot be read directly from the spectra. Therefore the data were fitted in the following manner. First, the deconvoluted peak of a spectrum of free BsCM was modeled by a smoothing spline function as described in the appendix. Then we assumed that the peaks of the protein-inhibitor complexes differ only in their position on the mass scale and their amplitude but not in their shape. This allows the contribution of all four $\mathrm{BsCM} / \mathrm{BsCM}$-inhibitor species to be determined from the shape of the previously determined BsCM signal. For fitting the spectra, two additional parameters were needed, one to account for the baseline level and a scaling factor that accounts for the absolute intensity of each spectrum. Since all binding sites are equal and independent and therefore do not show any allosteric or cooperative effect [16], only a single fitting parameter, a probability $\mathrm{P}$ that describes the occupation of a binding site by an inhibitor, fully determines the ratio of all four peaks (for details, see appendix).

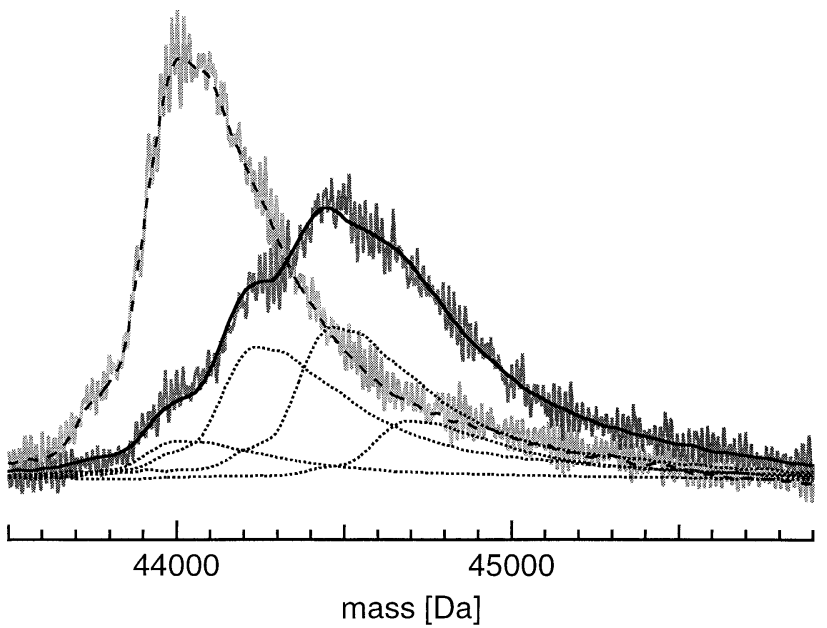

Figure 5. Representative figure of the fitted mass spectra (for details see text and appendix). The dashed line represents the function fitted to the deconvoluted mass spectrum of bare BsCM. The dotted lines are this function shifted by the inhibitor mass zero, one, two, or three times. The solid line is the sum of these four functions fitted to the deconvoluted spectra of BsCM plus 10 $\mu \mathrm{M}$ TSA. BsCM concentration was $3.3 \mu \mathrm{M}$.

As can be seen in Figure 5, which depicts an example of such a fit, the raw data are matched very well. At higher inhibitor concentrations, starting from $50 \mu \mathrm{M}$ which corresponds to a 15 -fold excess, the fits were less good, probably due to the onset of unspecific binding of inhibitor to the enzyme. Therefore only data for inhibitor concentrations below $50 \mu \mathrm{M}$ were used to calculate the $K_{d}$ value. The fitting parameter affords the relative peak areas for BsCM, BsCM·I, BsCM·I2, BsCM $\cdot \mathrm{I}_{3}$, allowing a dissociation constant to be calculated for each titration point as explained in the appendix. For the wild type enzyme BsCM and the TSA inhibitor a $\mathrm{K}_{\mathrm{d}}$ of $1.7 \pm 1 \mu M$ was determined, which compares well with the value of $1.0 \pm 0.2 \mu \mathrm{M}$ determined in the solutionphase assay. In the literature values between 1.7 [26] and $3 \mu \mathrm{M}$ [16] have been reported. For the truncated enzyme $\mathrm{BsCM}^{1-120}$ and TSA we determined $\mathrm{K}_{\mathrm{d}}$ to be 37 $\pm 6 \mu \mathrm{M}$ with our mass spectrometric method whereas a standard solution assay gave $25 \pm 1.7 \mu \mathrm{M}$. Again, the $\mathrm{K}_{\mathrm{d}}$ values determined by mass spectrometry and the solution-phase assay are in good agreement. Systematic errors can be introduced if the actual initial enzyme concentration used for the measurements is lower than stated, due to losses in the desalting process or precipitation while freezing and thawing the protein solutions.

\section{Conclusions}

Broad peaks are often a problem when measuring noncovalent complexes by ESI-MS. Since it is necessary to maintain the native structure of the protein for substrate or inhibitor binding, the number of charges loaded on the protein is usually low, leading to high $\mathrm{m} / \mathrm{z}$ values where the instrumental mass resolution drops. 
In addition, the preservation of weak noncovalent interactions necessitates the use of very soft desolvation conditions in the ESI source which leads to a further increase in peak width due to adducts. In this study, we overcame this problem by modeling the signal of noncovalent protein-inhibitor complexes as the sum of four single peaks derived from the signal of the "naked" (trimeric) enzyme. In doing so we obtained dissociation constants for BsCM and TSA as well as for BsCM ${ }^{1-120}$ and TSA. The absolute $K_{d}$ values determined by MS are in good agreement with solution-phase data.

\section{Acknowledgments}

The authors gratefully acknowledge the assistance of Dr. Reto Häring in the development of the algorithm for the data evaluation. They thank Rosalino Pulido for the synthesis of the TSA inhibitor and Stefan van Sint Fiet for construction of plasmid pAK-L121*. They also acknowledge with much appreciation the financial support of this research by Swiss National Science Foundation (Grants no. 2000-058861 and 3100-053743).

\section{References}

1. Beck, J. L.; Colgrave, M. L.; Ralph, S. F.; Sheil, M. M. Electrospray Ionization Mass Spectrometry of Oligonucleotide Complexes with Drugs, Metals, and Proteins. Mass Spectrom. Rev. 2001, 20, 61.

2. Loo, J. A. Studying Noncovalent Protein Complexes by Electrospray Ionization Mass Spectrometry. Mass Spectrom. Rev. 1997, 16, 1-23.

3. Loo, J. A. Electrospray Ionization Mass Spectrometry: A Technology for Studying Noncovalent Macromolecular Complexes. Int. J. Mass Spectrom. 2000, 200, 175-186.

4. Daniel, J. M.; Friess, S. D.; Rajagopalan, S.; Wendt, S.; Zenobi, R. Quantitative Determination of Noncovalent Binding Interactions Using Soft Ionization Mass Spectrometry. Int. J. Mass Spectrom. 2002, 216, 1-27.

5. Fändrich, M.; Tito, M. A.; Leroux, M. R.; Rostom, A. A.; Hartl, F. U.; Dobson, C. M.; Robinson, C. V. Observation of the Noncovalent Assembly and Disassembly Pathways of the Chaperone Complex MtGimC by Mass Spectrometry. Proc. Natl. Acad. Sci. U.S.A. 2000, 97, 14151-14155.

6. Loo, J. A.; Hu, P.; McConnell, P.; Mueller, W. T.; Sawyer, T. K.; Thanabal, V. A Study of Src SH2 Domain Protein-Phosphopeptide Binding Interactions by Electrospray Ionization Mass Spectrometry. J. Am. Soc. Mass Spectrom. 1997, 8, 234243.

7. Li, Y.-T.; Hsieh, Y.-L.; Henion, J. D.; Ocain, T. D.; Schiehser, G. A.; Ganem, B. Analysis of the Energetics of Gas-Phase Immunophilin-Ligand Complexes by Ion Spray Mass Spectrometry. J. Am. Chem. Soc. 1994, 116, 7487-7493.

8. Robinson, C. V.; Chung, E. W.; Kragelund, B. B.; Knudsen, J.; Aplin, R. T.; Poulsen, F. M.; Dobson, C. M. Probing the Nature of Noncovalent Interactions by Mass Spectrometry. A Study of Protein-CoA Ligand Binding and Assembly. J. Am. Chem. Soc. 1996, 118, 8646-8653.

9. Lim, H.-K.; Hsieh, Y. L.; Ganem, B.; Henion, J. Recognition of Cell-Wall Peptide Ligands by Vancomycin Group Antibiotics: Studies Using Ion Spray Mass Spectrometry. J. Mass Spectrom. 1995, 30, 708-714.

10. Greig, M. J.; Gaus, H.-J.; Cummins, L. L.; Sasmor, H.; Griffey, R. H. Measurement of Macromolecular Binding Using Electrospray Mass Spectrometry. Determination of Dissociation
Constants from Oligonucleotide-Serum Albumin Complexes. J. Am. Chem. Soc. 1995, 117, 10765-10766.

11. Ayed, A.; Krutchinsky, A. N.; Ens, W.; Standing, K. G.; Duckworth, H. W. Quantitative Evaluation of Protein-Protein and Ligand-Protein Equilibria of a Large Allosteric Enzyme by Electrospray Ionization Time-of-Flight Mass Spectrometry. Rapid Commun. Mass Spectrom. 1998, 12, 339-344.

12. Smith, R. D.; Bruce, J. E.; Wu, Q.; Lei, P. New Mass Spectrometric Methods for the Study of Noncovalent Associations of Biopolymers. Chem. Soc. Rev. 1997, 26, 192-202.

13. Griffey, R. H.; Sannes-Lowery, K. A.; Drader, J. J.; Mohan, V.; Swayze, E. E.; Hofstadler, S. A. Characterization of LowAffinity Complexes Between RNA and Small Molecules Using Electrospray Ionization Mass Spectrometry. J. Am. Chem. Soc. 2000, 122, 9933-9938.

14. Sannes-Lowery, K. A.; Griffey, R. H.; Hofstadler, S. A. Measuring Dissociation Constants of RNA and Aminoglycoside Antibiotics by Electrospray Mass Spectrometry. Anal. Biochem. 2000, 280, 264-271.

15. Chook, Y. M.; Ke, H. M.; Lipscomb, W. N. Crystal-Structures of the Monofuctional Chorismate Mutase from Bacillus subtilis and Its Complex with a Transition-State Analog. Proc. Natl. Acad. Sci. U.S.A. 1993, 90, 8600-8603.

16. Gray, J. V.; Even, D.; Knowles, J. R. Monofuctional Chorismate Mutase from Bacillus subtilis-Kinetic and C-13 NMR Studies on the Interactions of the Enzyme with Its Ligands. Biochemistry 1990, 29, 8872-8878.

17. Bartlett, P. A.; Nakagawa, Y.; Johnson, C. R.; Reich, S. H.; Luis, A. Chorismate Mutase Inhibitors-Synthesis and Evaluation of Some Potential Transition-State Analogs. J. Org. Chem. 1988, 53, 3195-3210.

18. Kast, P.; Grisostomi, C.; Chen, I. A.; Li, S.; Krengel, U.; Xue, Y.; Hilvert, D. A Strategically Positioned Cation is Crucial for Efficient Catalysis by Chorismate Mutase. J. Biol. Chem. 2000, 275, 36832-36838.

19. Mattei, P.; Kast, P.; Hilvert, D. Bacillus subtilis Chorismate Mutase is Partially Diffusion-Controlled. Eur. J. Biochem. 1999, 261, 25-32.

20. Sanger, F.; Nicklen, S.; Coulson, A. R. DNA Sequencing with Chain-Terminating Inhibitors. Proc. Natl. Acad. Sci. U.S.A. 1977, 74, 5463-5467.

21. MacBeath, G.; Kast, P.; Hilvert, D. A Small, Thermostable, and Monofunctional Chorismate Mutase from the Archaeon Methanococcus jannaschii. Biochemistry 1998, 37, 10062-10073.

22. Chao, H. S; Berchtold, G. A. Inhibition of Chorismate Mutase Activity of Chorismate Mutase-Prephenate Dehydrogenase from Aerobacter aerogenes. Biochemistry 1982, 21, 2778-2781.

23. Lemaire, D.; Marie, G.; Serani, L.; Laprevote, O. Stabilization of Gas-Phase Noncovalent Macromolecular Complexes in Electrospray Mass Spectrometry Using Aqueous Triethylammonium Bicarbonate Buffer. Anal. Chem. 2001, 73, 1699-1706.

24. Felitsyn, N.; Peschke, M.; Kebarle, P. Origin and Number of Charges Observed on Multiply-Protonated Native Proteins Produced by ESI. Int. J. Mass Spectrom. 2002, 219, 39-62.

25. Gamper, M.; Hilvert, D.; Kast, P. Probing the Role of the C-Terminus of Bacillus subtilis Chorismate Mutase by a Novel Random Protein-Termination Strategy. Biochemistry 2000, 39, 14087-14094.

26. Cload, S. T.; Liu, D. R.; Pastor, R. M.; Schultz, P. G. Mutagenesis Study of Active Site Residues in Chorismate Mutase from Bacillus subtilis. J. Am. Chem. Soc. 1996, 118, 1787-1788.

27. Reinsch, C. Smoothing by Spline Functions. Numer. Math. 1967, 10, 177-183.

28. Tanford, C. Physical Chemistry of Macromolecules; Wiley: New York, 1961. 


\section{Appendix}

\section{A. Modeling of the Deconvoluted Peak of the "Naked" Chorismate Mutase Trimer}

To model the shape of the deconvoluted peak of the "naked" trimer a function is needed which closely follows the data but rejects the noise. We used a "smoothing spline function" as described in reference [27] to achieve the smoothest curve that lies within the given tolerance of the measured data. Empirically, we found that a tolerance corresponding to $1 \%$ of the total amplitude gave satisfactory results for our data set.

\section{B. Calculating the Ratio of Peaks}

When binding sites are equal and independent as is the case for BsCM [16] we can define a probability $\mathrm{P}$ of a binding site being occupied by an inhibitor $(0<\mathrm{P}<$ $100 \%)$. The concentration of the various enzyme-inhibitor complexes can then be expressed as

$$
\begin{aligned}
& {[\mathrm{BsCM}]=(1-\mathrm{P})^{3} *[\mathrm{BsCM}]_{0}} \\
& {[\mathrm{BsCM} \cdot \mathrm{I}]=3 *(1-\mathrm{P})^{2} * \mathrm{P} *[\mathrm{BsCM}]_{0}} \\
& {\left[\mathrm{BsCM} \cdot \mathrm{I}_{2}\right]=3 *(1-\mathrm{P}) * \mathrm{P}^{2} *[\mathrm{BsCM}]_{0}} \\
& {\left[\mathrm{BsCM} \cdot \mathrm{I}_{3}\right]=\mathrm{P}^{3} *[\mathrm{BsCM}]_{0}}
\end{aligned}
$$

where the factor 3 accounts for the statistical permutations of the complex. Since $[\mathrm{BsCM}]_{0}$, the total enzyme concentration, is known, the entire complex distribution is defined by P. In other words, only a single fitting parameter describes the relative peak areas.

\section{Calculating Dissociation Constants}

The dissociation constants were calculated based on the general theory of multiple equilibria using only peak ratios. For the three equilibria, three dissociation constants $\mathrm{K}_{\mathrm{i}}$ can be defined and expressed with the $\mathrm{K}_{\mathrm{d}}$ for binding to an individual site of the enzyme as follows [28]:

$$
\begin{aligned}
& \mathrm{K}_{1}=[\mathrm{I}] *[\mathrm{BsCM}] /[\mathrm{BsCM} \cdot \mathrm{I}]=1 / 3 * \mathrm{~K}_{\mathrm{d}} \\
& \mathrm{K}_{2}=[\mathrm{I}] *[\mathrm{BsCM} \cdot \mathrm{I}] /\left[\mathrm{BsCM} \cdot \mathrm{I}_{2}\right]=\mathrm{K}_{\mathrm{d}} \\
& \mathrm{K}_{3}=[\mathrm{I}] *\left[\mathrm{BsCM} \cdot \mathrm{I}_{2}\right] /\left[\mathrm{BsCM} \cdot \mathrm{I}_{3}\right]=3 * \mathrm{~K}_{\mathrm{d}}
\end{aligned}
$$

where [I] is the free inhibitor concentration and the peak ratios are given by

$$
\begin{aligned}
& {[\mathrm{BsCM}] /[\mathrm{BsCM} \cdot \mathrm{I}]=(1-\mathrm{P}) /(3 * \mathrm{P})} \\
& {[\mathrm{BsCM} \cdot \mathrm{I}] /\left[\mathrm{BsCM} \cdot \mathrm{I}_{2}\right]=(1-\mathrm{P}) / \mathrm{P}} \\
& {\left[\mathrm{BsCM} \cdot \mathrm{I}_{2}\right] /\left[\mathrm{BsCM}^{\mathrm{B}} \cdot \mathrm{I}_{3}\right]=3 *(1-\mathrm{P}) / \mathrm{P}}
\end{aligned}
$$

where $\mathrm{P}$ is obtained from the fit of the data.

The total concentrations of the enzyme, $[\mathrm{BsCM}]_{0}$, and of the inhibitor, $\left[\mathrm{I}_{0}\right]$, are given by

$$
\begin{gathered}
{[\mathrm{BsCM}]_{0}=[\mathrm{BsCM}]+[\mathrm{BsCM}]+[\mathrm{BsCM} \cdot \mathrm{I}]} \\
+\left[\mathrm{BsCM} \cdot \mathrm{I}_{2}\right]+\left[\mathrm{BsCM} \cdot \mathrm{I}_{3}\right] \\
{\left[\mathrm{I}_{0}\right]=[\mathrm{I}]+[\mathrm{BsCM} \cdot \mathrm{I}]+2 *\left[\mathrm{BsCM} \cdot \mathrm{I}_{2}\right]} \\
+3 *\left[\mathrm{BsCM} \cdot \mathrm{I}_{3}\right]
\end{gathered}
$$

By inserting eqs 8,9 , and 10 into eq 11 and solving for [BsCM], and inserting eqs 8, 9, and 10 into eq 12 and solving for [I] one gets

$$
[\mathrm{I}]=\left[\mathrm{I}_{0}\right]-[\mathrm{BsCM}]_{0} * 3 \mathrm{P}
$$

By inserting eqs 9 and 13 into eq $6, K_{d}$ can be calculated from one single measurement. $K_{d}$ was determined for seven titration points in the range from 0 to $50 \mu \mathrm{M}$ TSA where satisfactory fits were obtained. From these seven $\mathrm{K}_{\mathrm{d}}$ values the standard deviation was calculated. 